\title{
The Periodic Cauchy Kernel, the Periodic Bochner Kernel, and Discrete Pseudo-Differential Operators
}

\author{
Vladimir Vasilyev \\ Chair of Pure Mathematics, Lipetsk State Technical University, Moskovskaya 30, Lipetsk 398600, Russia \\ vladimir.b.vasilyev@gmail.com
}

\begin{abstract}
We introduce a discrete pseudo-differential operator in an appropriate discrete functional space and study the invertibility properties for such simplest operators in certain canonical domains of an Euclidean space. We construct special projectors for studying these operators according to a type of canonical domain and show how these operators are related to special boundary value problems for holomorphic functions of several variables.
\end{abstract}

\section{INTRODUCTION}

A classical pseudo-differential operator in the Euclidean space $\mathbb{R}^{m}$ is defined by $([1,2])$

$$
(A u)(x)=\int_{\mathbb{R}^{m}} \int_{\mathbb{R}^{m}} \tilde{A}(x, \xi) e^{i(\xi-y)} \tilde{u}(\xi) d \xi d y,
$$

where the sign $\sim$ over a function denotes its Fourier transform

$$
\widetilde{u}(\xi)=\int_{\mathbb{R}^{m}} u(x) e^{i x \xi \xi} d x .
$$

Given a function $u_{d}$ of a discrete variable $\tilde{x} \in \mathbb{Z}^{m}$, we define its discrete Fourier transform by the series

$$
\left(F_{d} u_{d}\right)(\xi) \equiv \widetilde{u}_{d}(\xi)=\sum_{\tilde{x} \in \mathbb{Z}^{m}} e^{i \tilde{x} \xi} u(\tilde{x}), \quad \xi \in \mathbb{T}^{m},
$$

where the partial sums are taken over cubes

$$
Q_{N}=\left\{\tilde{x} \in \mathbb{Z}^{m}: \tilde{x}=\left(\tilde{x}_{1}, \cdots, \tilde{x}_{m}\right), \max _{1 \leq k \leq m}\left|\tilde{x}_{k}\right| \leq N\right\} .
$$

Let $D \subset \mathbb{R}^{m}$ be a cone, $D_{d} \equiv D \cap \mathbb{Z}^{m}$, and $L_{2}\left(D_{d}\right)$ be a space of functions of a discrete variable defined on $D_{d}$, and $A(\tilde{x})$ be a given function of a discrete variable $\tilde{x} \in \mathbb{Z}^{m}$. We consider the operators

$$
\left(A_{d} u_{d}\right)(\tilde{x})=\int_{\mathbb{T}^{m}} \sum_{y \in D_{d}} e^{i(\tilde{y}-\tilde{x}) \xi \xi} \widetilde{A}(\xi) \tilde{u}_{d}(\xi) d \xi, \quad \tilde{x} \in D_{d},
$$

and introduce the function

$$
\widetilde{A}_{d}(\xi)=\sum_{\tilde{x} \in \mathbb{Z}^{m}} e^{i \tilde{x} \cdot \xi} A(\tilde{x}), \quad \xi \in \mathbb{T}^{m} .
$$

Definition 1. The function $\widetilde{A}_{d}(\xi)$ is called a symbol of the operator $A_{d}$, and this symbol is called an elliptic symbol if $\tilde{A}_{d}(\xi) \neq 0, \forall \xi \in \mathbb{T}^{m}$. 


\section{DISCRETE PSEUDO-DIFFERENTIAL OPERATORS}

Definition 2. The formula (1) defines a discrete pseudo-differential operator in the canonical domain $D_{d}$.

Example 1. If $K(x), x \in \mathbb{R}^{m} \backslash\{0\}$, is a Calderon-Zygmund kernel, then the corresponding operator is defined by [3]

$$
\left(K_{d} u_{d}\right)(\tilde{x})=\sum_{\tilde{y} \in \mathbb{Z}^{m}, \tilde{y} \neq \tilde{x}} K(\tilde{x}-\tilde{y}), \quad \tilde{x} \in \mathbb{Z}^{m} .
$$

Example 2. If a first order finite difference of a discrete variable $\tilde{x}_{k}$ is defined by

$$
\delta_{k} u_{d}(\tilde{x})=u_{d}\left(\tilde{x}_{k}+1\right)-u_{d}\left(\tilde{x}_{k}\right)
$$

then the discrete Laplacian is

$$
\left(\Delta_{d} u_{d}\right)(\tilde{x})=\sum_{k=1}^{m}\left(u_{d}\left(\tilde{x}_{k}+2\right)-2 u_{d}\left(\tilde{x}_{k}+1\right)+u_{d}\left(\tilde{x}_{k}\right)\right)
$$

and its symbol is the function

$$
\sigma_{\Delta d}(\xi)=\sum_{k=1}^{m}\left(e^{i \xi_{k}}-1\right)^{2}
$$

\section{Discrete Sobolev-Slobodetskii Spaces}

Let $H^{s}\left(\mathbb{Z}^{m}\right)$ denotes the space of functions of a discrete variable for which

$$
\left\|u_{d}\right\|_{s}^{2} \equiv \int_{\mathbb{T}^{m}}\left|\widetilde{u}_{d}(\xi)\right|^{2}\left(1+\left|\sigma_{\Delta_{d}}(\xi)\right|\right)^{s} d \xi<+\infty
$$

We say a discrete operator $A_{d}$ has order $\alpha$ if its symbol $\widetilde{A}_{d}(\xi)$ satisfies the condition

$$
\left|\widetilde{A_{d}}(\xi)\right| \sim\left(1+\left|\sigma_{\Delta_{d}}(\xi)\right|\right)^{\frac{\alpha}{2}} .
$$

The class of such symbols will be denoted by $S_{\alpha}\left(\mathbb{T}^{m}\right)$.

Lemma 1. Pseudo-differential operator $A_{d}$ of order $\alpha$ is a linear bounded operator $H^{s}\left(\mathbb{Z}^{m}\right) \rightarrow H^{s-\alpha}\left(\mathbb{Z}^{m}\right)$.

Indeed,

$$
\left\|A_{d} u_{d}\right\|_{s}^{2}=\int_{\mathbb{T}^{m}}\left|\widetilde{A}_{d}(\xi) \widetilde{u}_{d}(\xi)\right|^{2}\left(1+\left|\sigma_{\Delta_{d}}(\xi)\right|\right)^{s} d \xi \leq c \int_{\mathbb{T}^{m}}\left|\widetilde{u}_{d}(\xi)\right|^{2}\left(1+\left|\sigma_{\Delta_{d}}(\xi)\right|\right)^{s+\alpha} d \xi .
$$

\section{DISCRETE PROJECTORS AND COMPLEX VARIABLES}

Let us denote by $P_{D_{d}}$ the projection operator on $D_{d}, P_{D_{d}}: L_{2}\left(\mathbb{Z}^{m}\right) \rightarrow L_{2}\left(D_{d}\right)$, so that for an arbitrary function $u_{d} \in L_{2}\left(\mathbb{Z}^{m}\right),\left(P_{D_{d}} u_{d}\right)(\tilde{x})=u_{d}(\tilde{x})$ if $\tilde{x} \in D_{d}$, and $u_{d}(\tilde{x})=0$ otherwise.

\section{Half-Space Case and Periodic Cauchy kernel}

If we consider a half-space case, then the Fourier image of the operator $P_{D_{d}}$ can be evaluated $([3,4])$ and we'll demonstrate it in the following

Example 3. If $D=\mathbb{R}_{+}^{m}$, then

$$
\left(F_{d} P_{D_{d}} u_{d}\right)\left(\xi^{\prime}, \xi_{m}\right)=\frac{1}{4 \pi i} \lim _{\tau \rightarrow 0+} \int_{-\pi}^{\pi} u_{d}\left(\xi^{\prime}, \eta_{m}\right) \cot \frac{\xi_{m}-\eta_{m}+i \tau}{2} d \eta_{m} .
$$


Thus we use a periodic one-dimensional Riemann problem with a parameter $\xi^{\prime} \in \mathbb{T}^{m-1}$ which is the following. Finding a pair of functions $\Phi^{ \pm}\left(\xi^{\prime}, \xi_{m}\right)$ which are boundary values of holomorphic functions in half-strips $\Pi_{ \pm}=\{z \in$ $\left.\mathbb{C}: z=\xi_{m} \pm i \tau, \tau>0\right\}$ such that these are satisfied a linear relation

$$
\Phi^{+}(\xi)\left(\xi^{\prime}, \xi_{m}\right)=G\left(\xi^{\prime}, \xi_{m}\right) \Phi^{-}(\xi)\left(\xi^{\prime}, \xi_{m}\right)+g(\xi), \quad \xi \in \mathbb{T}^{m},
$$

for almost all $\xi^{\prime} \in \mathbb{T}^{m-1}$, where $G(\xi), g(\xi)$ are given periodic functions. It looks like the classical cases $[5,6]$.

\section{Conical Case and Periodic Bochner kernel}

Let $D$ be a sharp convex cone, and $\dot{D}$ be a conjugate cone for $D$, i.e.,

$$
\stackrel{*}{D}=\left\{x \in \mathbb{R}^{m}: x \cdot y>0, y \in D\right\} .
$$

Let $T(\dot{D}) \subset \mathbb{C}^{m}$ be a set of the type $\mathbb{T}^{m}+i \dot{D}$. For $\mathbb{T}^{m} \equiv \mathbb{R}^{m}$ such a domain of multidimensional complex space is called a radial tube domain over the cone $\stackrel{*}{D}([7,8,9])$. We introduce the function

$$
B_{d}(z)=\sum_{\tilde{x} \in D_{d}} e^{i \tilde{x} z}, \quad z=\xi+i \tau, \quad \xi \in \mathbb{T}^{m}, \quad \tau \in \stackrel{*}{D},
$$

and define the operator

$$
\left(B_{d} u\right)(\xi)=\lim _{\tau \rightarrow 0} \int_{\mathrm{T}^{m}} B_{d}(z-\eta) u_{d}(\eta) d \eta .
$$

Lemma 2. For arbitrary $u_{d} \in L_{2}\left(\mathbb{Z}^{m}\right)$, the following property

$$
F_{d} P_{D_{d}} u_{d}=B_{d} F_{d} u_{d}
$$

holds.

Let us define the subspace $A\left(\mathbb{T}^{m}\right) \subset L_{2}\left(\mathbb{T}^{m}\right)$ consisting of functions which admit a holomorphic continuation into $T(\stackrel{*}{D})$ and satisfy the condition

$$
\sup _{\tau \in D} \int_{\mathbb{T}^{m}}\left|\tilde{u}_{d}(\xi+i \tau)\right|^{2} d \xi<+\infty
$$

In other words, the space $A\left(\mathbb{T}^{m}\right) \subset L_{2}\left(\mathbb{T}^{m}\right)$ consists of boundary values of holomorphic in $T(D)$ functions.

Let us denote

$$
B\left(\mathbb{T}^{m}\right)=L_{2}\left(\mathbb{T}^{m}\right) \ominus A\left(\mathbb{T}^{m}\right),
$$

so that $B\left(\mathbb{T}^{m}\right)$ is a direct complement of $A\left(\mathbb{T}^{m}\right)$ in $L_{2}\left(\mathbb{T}^{m}\right)$.

\section{A jump problem}

We formulate the problem in the following way: finding a pair of functions $\Phi^{ \pm}, \Phi^{+} \in A\left(\mathbb{T}^{m}\right), \Phi^{-} \in B\left(\mathbb{T}^{m}\right)$, such that

$$
\Phi^{+}(\xi)-\Phi^{-}(\xi)=g(\xi), \quad \xi \in \mathbb{T}^{m},
$$

where $g(\xi) \in L_{2}\left(\mathbb{T}^{m}\right)$ is given.

Lemma 3. The operator $B_{d}: L_{2}\left(\mathbb{T}^{m}\right) \rightarrow A\left(\mathbb{T}^{m}\right)$ is a bounded projector. A function $u_{d} \in L_{2}\left(D_{d}\right)$ iff its Fourier transform $\tilde{u}_{d} \in A\left(\mathbb{T}^{m}\right)$.

Theorem 1. The jump problem has unique solution for arbitrary right-hand side from $L_{2}\left(\mathbb{T}^{m}\right)$.

Example 4. If $m=2$ and $D$ is the first quadrant in a plane then a solution of a jump problem is given by formulas

$$
\begin{gathered}
\Phi^{+}(\xi)=\frac{1}{(4 \pi i)^{2}} \lim _{\tau \rightarrow 0} \int_{-\pi}^{\pi} \int_{-\pi}^{\pi} \cot \frac{\xi_{1}+i \tau_{1}-t_{1}}{2} \cot \frac{\xi_{2}+i \tau_{2}-t_{2}}{2} g\left(t_{1}, t_{2}\right) d t_{1} d t_{2} \\
\Phi^{-}(\xi)=\Phi^{+}(\xi)-g(\xi), \quad \tau=\left(\tau_{1}, \tau_{2}\right) \in D .
\end{gathered}
$$


It looks as follows. Finding a pair of functions $\Phi^{ \pm}, \Phi^{+} \in A\left(\mathbb{T}^{m}\right), \Phi^{-} \in B\left(\mathbb{T}^{m}\right)$, such that

$$
\Phi^{+}(\xi)=G(\xi) \Phi^{-}(\xi)+g(\xi), \quad \xi \in \mathbb{T}^{m}
$$

where $G(\xi), g(\xi)$ are given periodic functions. If $G(\xi) \equiv 1$, we have the jump problem (2). (3).

Like classical studies $[5,6]$, we want to use a special representation for an elliptic symbol to solve the problem

\section{Periodic wave factorization}

Let us denote $H^{s}\left(D_{d}\right)$ a subspace of $H^{s}\left(\mathbb{Z}^{m}\right)$ consisting of functions of discrete variable $\tilde{x}$ for which their supports belong to $\overline{D_{d}}$, and $\widetilde{H}^{s}\left(D_{d}\right), \widetilde{H}^{s}\left(\mathbb{Z}^{m}\right)$ their Fourier images.

Lemma 4. For $|s|<1 / 2$, the operator $B_{d}$ is a bounded projector $\widetilde{H}^{s}\left(\mathbb{Z}^{m}\right) \rightarrow \widetilde{H}^{s}\left(D_{d}\right)$, and a jump problem has unique solution $\Phi^{+} \in \widetilde{H}^{s}\left(D_{d}\right), \Phi^{-} \in \widetilde{H}^{s}\left(\mathbb{Z}^{m} \backslash D_{d}\right)$ for arbitrary $g \in \widetilde{H}^{s}\left(\mathbb{Z}^{m}\right)$.

Definition 3. Periodic wave factorization for elliptic symbol $\tilde{A}(\xi)$ is called its representation in the form

$$
\tilde{A}_{d}(\xi)=\tilde{A}_{\neq}(\xi) \tilde{A}_{=}(\xi)
$$

where the factors $A_{\neq}^{ \pm 1}(\xi), A_{=}^{ \pm 1}(\xi)$ admit bounded holomorphic continuation into domains $T( \pm \stackrel{*}{D})$.

Theorem 2. If $|s|<1 / 2$ and the elliptic symbol $\tilde{A}_{d}(\xi) \in S_{\alpha}\left(\mathbb{T}^{m}\right)$ admits periodic wave factorization, then the operator $A_{d}$ is invertible in the space $H^{s}\left(D_{d}\right)$.

\section{CONCLUSION}

These considerations have to be useful for statements of boundary value problems for discrete elliptic pseudodifferential equations in canonical non-smooth domains. Such boundary value problems will appear when an index of the wave factorization is not zero. Moreover we hope to establish a correspondence between discrete and continual [9] cases and to describe a limit transfer from discrete case to continual one.

\section{ACKNOWLEDGMENTS}

This work was partially supported by Russian Foundation for Basic Research and Lipetsk regional government of Russia, project no. 14-41-03595-r-center-a.

\section{REFERENCES}

[1] M. Taylor, Pseudodifferential Operators (Princeton Univ. Press, Princeton, NJ, 1981).

[2] G. Eskin, Boundary Value Problems for Elliptic Pseudodifferential Equations (AMS, Providence, RI, 1981).

[3] A. Vasilyev and V. Vasilyev, Azerb. J. Math. 3, 84-93 (2013).

[4] A. Vasilyev and V. Vasilyev, Differential Equat. 51, 652-660 (2015).

[5] F. Gakhov, Boundary Value Problems (Dover Publications, Mineola, NY, 1981).

[6] N. Muskhelishvili, Singular Integral Equations (North Holland, Amsterdam, 1976).

[7] S. Bochner and W. Martin, Several Complex Variables (Princeton Univ. Press, Princeton, NJ, 1948).

[8] V. Vladimirov, Methods of the Theory of Functions of Many Complex Variables (Dover Publications, Mineola, NY, 2007).

[9] V. Vasil'ev, Wave Factorization of Elliptic Symbols: Theory and Applications (Kluwer Academic Publishers, Dordrect-Boston-London, 2000). 